\title{
A Fractional Calculus Approach to Modeling Fractal Dynamic Games
}

\author{
Paul Bogdan and Radu Marculescu
}

\begin{abstract}
Motivated by the complexity of spatio-temporal patterns of interconnected human processes (e.g., crowds, car traffic, social networks), this paper sets forth the fractal dynamic games as an analytical tool for modeling and predicting human dynamics. Starting from a statistical physics description of interactions between agents and from the observed statistical properties of economic measures, we construct a master equation characterizing the dynamics of cost functionals as stochastic variables affected by additive and multiplicative noise forces. Given the significance of human behavior, we allow the cost distribution to depend on the evolution of agents density. By coupling the description of agent dynamics through a fractal structure with a generic stochastic utility function, we formulate a new dynamic game. Employing optimal control theory concepts, we derive a continuum formulation of the car traffic dynamics optimization resulting in a nonlinear fractional partial differential equation.
\end{abstract}

\section{INTRODUCTION AND PRIOR WORK}

The study of the dynamics of coupled technological and human networks has received significant attention in recent years. On the one hand, this is the result of major theoretical developments in statistical physics via network science [2][3], economics via game theory [15][16][25][26] and econometrics [32]; on the other hand, it is the outcome of the highly increasing availability of large data sets and computational processing power for information storage, analysis and mining. As far as these latter aspects are concerned, we have witnessed research efforts to quantify the statistical characteristics of various processes. These efforts were brought to fruition when Mandelbrot developed the fractal theory [31]. From heart rate signals [20] to brain activity [42], from price volatility to individual wealth [6], from communication workload over off [29] and on-chip [7] links to electron transportation in semiconductor devices, all processes exhibit fractal properties.

By employing network theory concepts researchers were able to distinguish between Erdos-Renyi and scale-free graphs, as well as characterize the structure of networks such as the Internet, social networks, biological networks, epidemiological networks, etc. For instance, Barabasi and Albert [2] have shown that the structure of genetic networks and the World Wide Web are better described by power laws than by Poisson distribution. Along the same lines, Blank and

This work was supported by the NSF, under Grant CCF-0916752. The work of P. Bogdan was supported in part through a fellowship from the Roberto Rocca Education Program.

P. Bogdan is with Electrical and Computer Engineering Department, Carnegie Mellon University, 5000 Forbes Ave., Pittsburgh, PA 15213, USA pbogdan@ece.cmu.edu

$\mathrm{R}$, Marculescu is with Electrical and Computer Engineering Department, Carnegie Mellon University, 5000 Forbes Ave., Pittsburgh, PA 15213, USA radumeece. cmu.edu
Solomon [6] have proposed a power law model for calculating the distribution of city population and investors' wealth on stock markets. All these findings pose new challenges to modeling networked human processes.

Concerned with modeling competition and coordination behavior in interconnected human processes, game theory approaches [34][33][30][37][1] have evolved into evolutionary game theory [11][19][12][40]. Evolutionary games are defined as ordinary differential equations of some macroscopic measures (e.g., mean expected success of a strategy). Along the same lines, Helbing [15][16] proposed a microscopic view of evolutionary dynamic games by describing individual behavior via a classical master equation [35][14]. In this setup, each individual can choose one strategy from a set of given strategies and the master equation describes the dynamics of the probability of finding a certain configuration of individuals embracing the set of strategies.

By taking the theory of dynamical games to a new level and relying on statistical mechanics principles, Lasry and Lions [25][26][27][28] set forth the concept of mean field games (MFG). In this context, the individuals in a population group interact with each other like particles in a thermodynamic gas and their state is described by a stochastic differential equation. The state and action of each individual affect the instantaneous cost function of all other players through a statistical distribution of the individuals [22]. Simply speaking, the aim of MFG is to minimize a cost function associated with a population of players. Hence, it depends on the distribution of players dynamics. This distribution is defined via a Fokker-Planck equation.

Several applications of MFG have been discussed in the context of the production of an exhaustible resource [23], human capital accumulation [28], pedestrian dynamics [10], and in the choice of an insulation technology in a group of households [24]. Motivated by the numerical analysis of MFG, a discrete time finite state space description has been presented in [21]. Along the same lines, Friedman and Ostrov [13] show that the dynamics of players on a financial market can exhibit shock waves leading to a clumped distribution. In simple terms, their conclusion is that the interactions among agents (e.g., adjustments of costs) dictated also by the inter-play between population structures or distribution and landscape lead to nontrivial dynamics.

Despite the considerable contribution of evolutionary game theory and mean field games, which has complemented prior advances in the field of statistical mechanics and game theory, we argue that one needs to take into account the intrinsic space and time characteristics (e.g., power law inter-event times between individual actions or price 

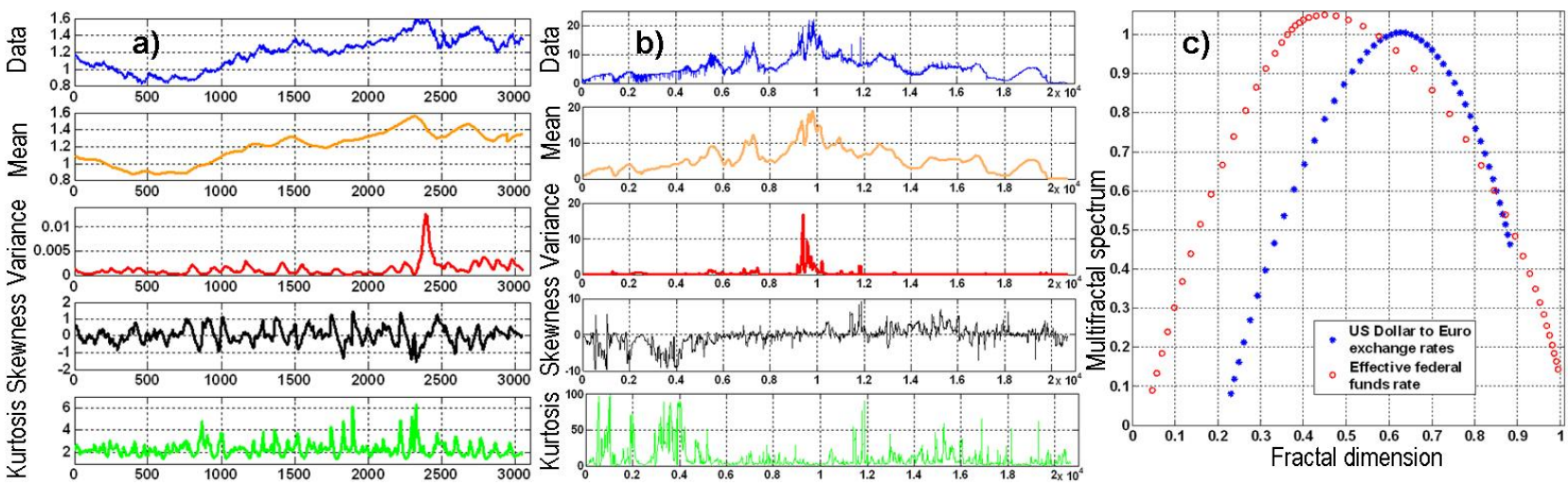

Fig. 1. a) US Dollar to Euro exchange rates between January 4th, 1999 and February 11th, 2011 exhibits a time dependent mean, variance, skewness and kurtosis. b) Time series of the effective federal funds rate between July 1st, 1954 and February 16th, 2011 also exhibit a non-stationary statistical behavior. c) Multifractal spectrum for of US Dollar to Euro exchange rates and effective federal funds rates which influence the players costs.

changes) of interconnected human processes for modeling and optimization purposes. In this context, we set forth a fractal dynamic game as a finite horizon optimization of two coupled equations: a fractional Fokker-Planck equation describing the dynamics of the density of agents through a fractal structure and a fractional master equation governing the cost evolution (which depends on agents density). To illustrate such a dynamic game, we consider the problem of traffic modeling where agents represent cars on fractal roads.

The remainder of the paper is structured as follows. In Section II.A, we discuss how the master equation of cost variable, which is subject to additive and multiplicative noise forces, can be constructed. Having the cost characterized via a master equation helps us choose a better optimization function in terms of higher order moments. Section II.B describes how the agents dynamics through fractal structures can be modeled. For completeness, we also relate various model parameters to real world measurements (i.e. car flow). Section III provides the mathematical description of the fractal dynamic game and the derivation of a fractal Hamilton-Jacoby equation. Section IV summarizes the paper contribution and gives a survey of future work.

\section{FRACTAL-BASED TRAFFIC MODELING}

\section{A. A Statistical Physics Approach to Game Utility Modeling}

Like any complex system, the macroscopic perspective of traffic dynamics and congestion reveals that car traffic exhibits various characteristics such as competition, frustration, collectivity (collective behavior), adaptability, sporadic synchronization, pseudo-periodicity, complex space and time correlations. From a microscopic perspective, the traffic is understood as a complex phenomenon that results from the effort of each agent (particle) that travels from one particular source point to a very specific destination while seeking to optimize a cost function (e.g., time of total traveling from source to destination, amount of money/fuel spent/consumed during the entire itinerary). This led towards a game theory approach where each agent optimizes a deterministic cost function [25][26][27][28].

However, due to the complex nature of human behavior and the availability of advanced technology in a large number of current vehicles (e.g., satelite radio/television that allow the quick spread of news about gas price, sale events, jammed roads, etc.), the cost cannot be reduced to a deterministic function. In addition to being stochastic in nature, many of such costs exhibit complex statistical and time dependent characteristics. Fig. 1 shows the time series and a few statistical measures (i.e., mean, variance, skewness and kurtosis) of the US Dollar to Euro exchange rates (a) and the effective federal fund rates (b) over a certain time interval. These time series exhibit not only a pronounced non-stationary signature, but also non-zero higher order moments. This implies that the modeling of these real world processes should not rely on Gaussian assumptions. In addition, many real world processes exhibit a dynamics that can be characterized by one or multiple fractal dimensions, as shown in Fig. 1.c. Given this fact, modeling approaches need to embrace the use of fractional derivatives [18]. Consequently, our next goal is to show how we can construct a dynamical equation based on fractional derivatives to model a process exhibiting fractal behavior.

In addition to the reported economic metrics, there are many other factors that affect human behavior. For instance, although one agent seeks to minimize his/her amount of money spent on gas by driving along the shortest path, a sales event news or a nearby accident along its predefined shortest path may make him/her choose alternative longer paths contributing to increasing his/her expenses not necessarily in an additive manner, but also in a multiplicative way. Simply speaking, we can represent the cost paid by an agent by means of the following stochastic differential equation:

$$
\frac{d^{\alpha} u(x, y, t)}{d t^{\alpha}}=f(u, d, t)+g(u, d, t) \xi(t)+\eta(t)
$$

where $u(x, y, t)$ defines the cost an agent pays to reach his/her destination and $\alpha$ is the fractional order of the derivative associated with the fractal behavior. In accordance with our assumption about various nonlinear interactions that contribute to a particular cost instance, (1) describes the fractal dynamics of the cost function $u(x, y, t)$, which is subject to an external field $V(u)$, where $f(u, d, t)=$ $-\frac{d V(u, d, t)}{d u}$ can be represented by a nonlinear dependence between the previous costs $u(x, y, t)$ and previous decisions $d(t)$, a weighted functional $g(u, d, t)$, multiplicative 

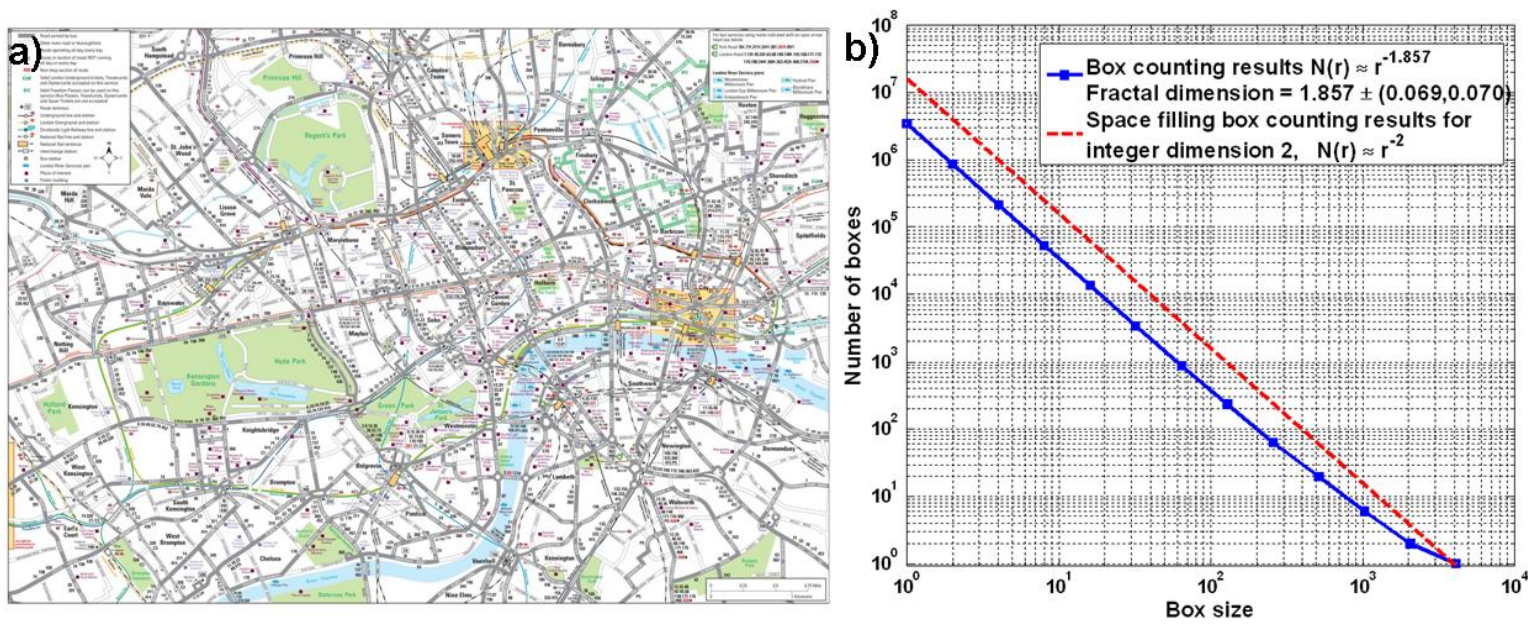

Fig. 2. a) Map of central London streets. b) Comparison between the number of boxes as a function of box size results for the street structure of London and a full two dimensional plane. The London streets configuration presents a fractal structure with a fractal dimension of 1.857.

noise $\xi(t)$ and additive noise $\eta(t)$. From a thermodynamic perspective, the existence of multiplicative noise can be justified by the stochastic nature of the external fields (e.g., goals, desideratum, news broadcasting, fuel price fluctuations, money income) or boundary conditions (e.g., salary increases, depreciation). Along the same lines, the additive noise can be seen as the result of the existence of various degrees of freedom choices (e.g., particular decisions can restrict or spread the amount of choices at later stages in the dynamics) or the thermal agitation (non-zero temperature) of the entire traffic system.

Adopting a statistical physics approach ${ }^{1}$, our next goal is to find a Fokker-Planck type of equation describing the dynamics of the probability distribution function (PDF) $P(u, t)$ that the stochastic cost variable attains value $u$ at time $t$ or in mathematical terms $P(u, t)=\langle\delta(u-u(t))\rangle$, where $\delta(u-u(t))$ represents the Delta function and the angular brakets $\langle\ldots\rangle$ represent the averaging over the noise realisations. Using the statistical physics standard method of characteristic function of cost $u(x, y, t)$, we compute the Fourier transform of $P(u, t)$ obtaining:

$$
P(v, t)=\int P(u, t) e^{-i v u} d u=\left\langle e^{-i v u(t)}\right\rangle
$$

Relying on the calculus of variations techniques, we construct a dynamical equation for the PDF $P(u, t)$ by computing the changes $\delta P(v, t)=P(v, t+\Delta t)-P(v, t)$ determined by an infinitesimal time increment $\Delta t \rightarrow 0$ :

$$
\begin{aligned}
& \delta P(v, t)=-i v \Delta t^{\alpha}\left\langle e^{-i v u(x, y, t)} f(u, d, t)\right\rangle+ \\
& +\left\langle e^{-i v u(x, y, t)}\left(e^{-i v g(u, d, t) \delta \xi}-1\right)\right\rangle+ \\
& +\left\langle e^{-i v u(x, y, t)}\left(e^{-i v \delta \eta}-1\right)\right\rangle
\end{aligned}
$$

where the term $\Delta t^{\alpha}$ is a consequence of the fact that the stochastic process $u(x, y, t)$ may exhibit fractal dynamics as shown in Figure 1.c. Using the properties of Fourier

\footnotetext{
${ }^{1}$ In statistical physics, the main tool for studying stochastic dynamics affected by multiplicative noise is by constructing a dynamical equation for the probability distribution function [9].
}

transform, the first term in equation (3) can be written as follows:

$$
\int i v e^{i v u}\left\langle e^{-i v u} f(u, d, t)\right\rangle d v=\frac{\partial[f(u, d, t) P(u, t)]}{\partial u}
$$

Moreover, we divide both sides of (3) by $\Delta t^{\alpha}$, take the limit $\Delta t \rightarrow 0$ and consider the following defition of fractional derivative of order $\alpha$ [18]:

$$
\frac{\partial^{\alpha} P(u, t)}{\partial t^{\alpha}}=\lim _{\Delta t \rightarrow 0} \frac{P(u, t+\Delta t)-P(u, t)}{\Delta t^{\alpha}}
$$

Hence, we obtain the next dynamical equation for $P(u, t)$ :

$$
\begin{aligned}
& \frac{\partial^{\alpha} P(u, t)}{\partial t^{\alpha}}=-\frac{\partial[f(u, d, t) P(u, t)]}{\partial u}+ \\
& +\lim _{\Delta t \rightarrow 0} \int \frac{p_{\xi}\left(\frac{u-z}{g(z, d, t)}, \Delta t\right)-\delta\left(\frac{u-z}{g(z, d, t)}\right)}{|g(z, d, t)| \Delta t^{\alpha}} P(z, t) d z+ \\
& +\lim _{\Delta t \rightarrow 0} \int \frac{p_{\eta}(u-z, \Delta t)-\delta(u-z)}{\Delta t^{\alpha}} P(z, t) d z
\end{aligned}
$$

Equation (6) describes the evolution of $P(u, t)$ associated with a fractal process $u$ via fractional derivatives rather than the classical integer order derivative. This is partially justified by the cost time series in Fig. 1. Although these series are continuous, they are not differentiable.

\section{B. Traffic Through Fractal Road Structures}

Statistical physics aims at enhancing our understanding of the properties and evolution of systems consisting of many interacting elements in both space and time [39]. Consequently, instead of dealing with and analyzing the evolution of each agent in our game (e.g., number of cars in road traffic, number of buyers on market), we assume that our entire population of agents is distributed across space and evolves according to very specific rules (depending on the nature of interactions). More precisely, we seek to describe the evolution of a PDF $n(x, y, t)$ (where $\iint n(x, y, t) d x d y=1$ for any time instant $t$ ) representing the density of agents at space coordinates $(x, y)$ at time $t$. For simplicity, we also assume that all agents have similar interests. This implies that agents try to minimize their cost function (e.g., traveling time, fuel consumption). 

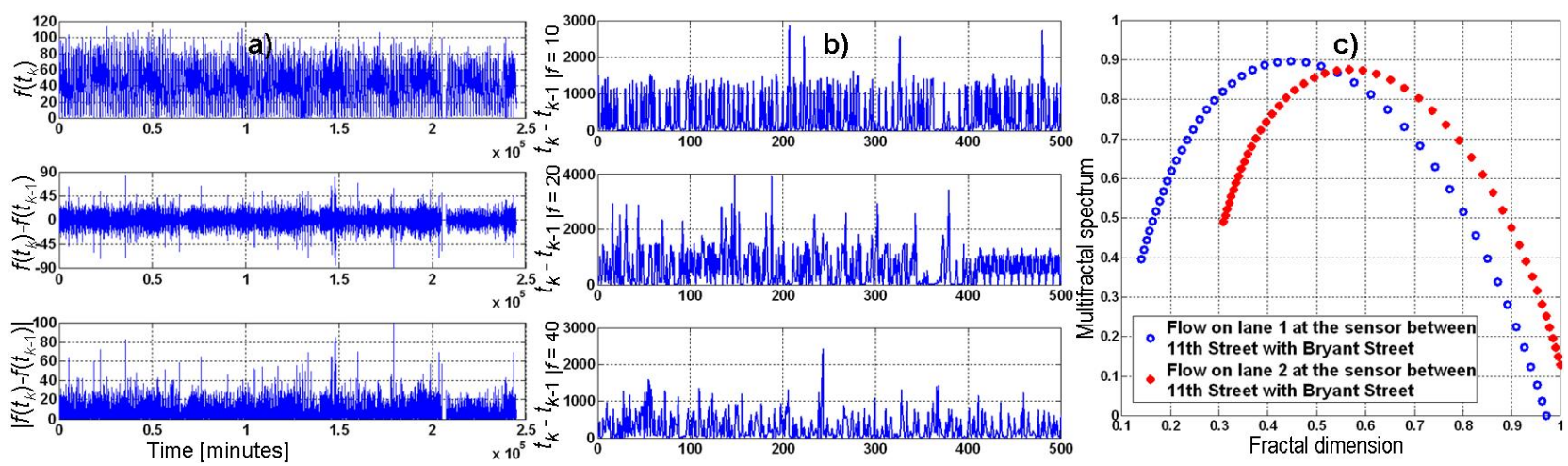

Fig. 3. a) Time series for the traffic flow, flow increments and absolute flow increments recorded between February 3, 2010 and February 22,2011 at the sensor between the 11th Street and Bryant Street in San Francisco obtained from [8]. Both the flow increments and their absolute values exhibit a self-similar structure. b) Time intervals at which the traffic flow attains value 10, 20, and 40 cars per 5 minutes exhibit a spiky behavior. c) The observed self-similar structure is confirmed by measuring the multifractal spectrum of the traffic flows along the two lanes recorded by the sensor. The existence of fractal behavior calls for new modeling approaches of road traffic.

Before going into the details of deriving a dynamical equation for $n(x, y, t)$, we make a few observations: 1$)$ the structure on which our agents (cars) evolve (move) does not entirely fill the $2 \mathrm{D}$ (or for that matter 3D) and is most often proved to be fractal [5]; 2) the distribution of agents across the entire space is not necessarily uniform, but it is frequently very skewed, maybe even power law distributed; 3) the distribution of inter-event times (e.g., time intervals between successive traffic flow increments) is better approximated by a power law rather than by an exponential distribution denoting a fractal dynamic of traffic processes. For completeness, Fig. 2 shows the road map of London (a) and the box counting ${ }^{2}$ graph leading to a fractal dimension of 1.857 . The existence of fractional dimensionality in road structures calls for modeling approaches based on fractional derivatives of the distribution of players (cars) in a dynamic game. In addition, Fig. 3 shows the traffic flow, flow increments and their multifractal spectrum. This calls for a new modeling approach to the agents density dynamics (i.e., $n(x, y, t)$ ).

To derive a partial differential equation for the density $n(x, y, t)$, we follow the classical technique of Wang and Uhlenbeck [41] and consider the following integral together with its continuum limit time derivative approximation:

$$
\begin{aligned}
& \iint R(x, y) \frac{\partial^{\beta} n(x, y, t)}{\partial t^{\beta}} d x d y= \\
& =\lim _{\Delta t \rightarrow 0} \iint R(x, y) \frac{n(x, y, t+\Delta t)-n(x, y, t)}{\Delta t^{\beta}} d x d y
\end{aligned}
$$

where $R(x, y)$ is an arbitrary function which vanishes for $x, y \rightarrow \pm \infty$. Note that in (7) we assume that the evolution of $n(x, y, t)$ is fractal in time as shown in Fig. 3 and use the definition in (5) to express the fractal dynamics via a fractional derivative of order $\beta$. Using the Bayes formula, the right hand side in (7) can be written as follows:

$$
\lim _{\Delta t \rightarrow 0} \iint R(x, y) \frac{n(x, y, t+\Delta t)-n(x, y, t)}{\Delta t^{\beta}} d x d y=
$$

\footnotetext{
${ }^{2}$ The box counting method is used to estimate the fractal dimension of an object as the slope of a linear fit between the logarithm of the number of boxes requires to cover optimally the object and the logarithm of the box size [31].
}

$$
\begin{aligned}
= & \lim _{\Delta t \rightarrow 0} \iint \frac{R(x, y)}{\Delta t^{\beta}}\left\{\iint n\left(x, y, t+\Delta t \mid x_{1}, y_{1}, t\right) .\right. \\
& \left.n\left(x_{1}, y_{1}, t\right) d x_{1} d y_{1}-n(x, y, t)\right\} d x d y
\end{aligned}
$$

Interchanging the order of integration and using the following fractional Taylor expansion:

$$
\begin{gathered}
R(x, y)=R\left(x_{1}, y_{1}\right)+\left.\left(x-x_{1}\right)^{\gamma} \frac{\partial^{\gamma} R(x, y)}{\partial x^{\gamma}}\right|_{\substack{(x, y)=\\
\left(x_{1}, y_{1}\right)}}+ \\
+\left.\left(y-y_{1}\right)^{\gamma} \frac{\partial^{\gamma} R(x, y)}{\partial y^{\gamma}}\right|_{\substack{(x, y)=\\
\left(x_{1}, y_{1}\right)}}+\left.\left(x-x_{1}\right)^{2 \gamma} \frac{\partial^{2 \gamma} R(x, y)}{\partial x^{2 \gamma}}\right|_{\substack{(x, y)=\\
\left(x_{1}, y_{1}\right)}} \\
+\left(x-x_{1}\right)^{\gamma}\left(y-y_{1}\right)^{\gamma}\left[\frac{\partial^{2 \gamma} R(x, y)}{\partial x^{\gamma} \partial y^{\gamma}}+\frac{\partial^{2 \gamma} R(x, y)}{\partial y^{\gamma} \partial x^{\gamma}}\right]_{\substack{(x, y)=\\
\left(x_{1}, y_{1}\right)}}+ \\
+\left.\left(y-y_{1}\right)^{2 \gamma} \frac{\partial^{2 \gamma} R(x, y)}{\partial y^{2 \gamma}}\right|_{\substack{(x, y)=\\
\left(x_{1}, y_{1}\right)}}+O\left(x^{2 \gamma}, y^{2 \gamma}\right)+\ldots
\end{gathered}
$$

the density $n(x, y, t)$ satisfies the following equation:

$$
\begin{gathered}
\iint R(x, y) \frac{\partial^{\beta} n(x, y, t)}{\partial t^{\beta}} d x d y= \\
=\iint n(x, y, t)\left[\left(x-x_{1}\right)^{\gamma} \frac{\partial^{\gamma} R}{\partial x^{\gamma}}+\left(y-y_{1}\right)^{\gamma} \frac{\partial^{\gamma} R}{\partial y^{\gamma}}+\right. \\
+\left(x-x_{1}\right)^{\gamma}\left(y-y_{1}\right)^{\gamma}\left(\frac{\partial^{2 \gamma} R}{\partial x^{\gamma} \partial y^{\gamma}}+\frac{\partial^{2 \gamma} R}{\partial y^{\gamma} \partial x^{\gamma}}\right)+ \\
\left.+\left(x-x_{1}\right)^{2 \gamma} \frac{\partial^{2 \gamma} R(x, y)}{\partial x^{2 \gamma}}+\left(y-y_{1}\right)^{2 \gamma} \frac{\partial^{2 \gamma} R}{\partial y^{2 \gamma}}\right] d x d y
\end{gathered}
$$

where $\gamma$ is the fractional order associated with the fractal structure on which the agents evolve. Considering the integration by parts formula:

$$
\int \frac{\partial^{\gamma} R(x, y)}{\partial x^{\gamma}} n(x, y, t) d x=\int R(x, y) \frac{\partial^{\gamma} n(x, y, t)}{\partial x^{\gamma}} d x
$$

(10) can now be written into the following form:

$$
\begin{gathered}
\iint R(x, y)\left\{\frac{\partial^{\beta} n(x, y, t)}{\partial t^{\beta}}-\frac{\partial^{\gamma}}{\partial x^{\gamma}}\left[A_{1}(x, y, t) n(x, y, t)\right]-\right. \\
-\frac{\partial^{\gamma}}{\partial y^{\gamma}}\left[A_{2}(x, y, t) n(x, y, t)\right]-\frac{\partial^{2 \gamma}}{\partial x^{2 \gamma}}\left[A_{3}(x, y) n(x, y, t)\right]- \\
-\frac{\partial^{2 \gamma}}{\partial y^{2 \gamma}}\left[A_{6}(x, y) n(x, y, t)\right]-\frac{\partial^{2 \gamma}}{\partial x^{\gamma} \partial y^{\gamma}}\left[A_{4}(x, y) n(x, y, t)\right] \\
\left.-\frac{\partial^{2 \gamma}}{\partial y^{\gamma} \partial x^{\gamma}}\left[A_{5}(x, y) n(x, y, t)\right]\right\} d x d y=0
\end{gathered}
$$

where internal functions $A_{1}(x, y, t), A_{2}(x, y, t), A_{3}(x, y, t)$, $A_{4}(x, y, t), A_{5}(x, y, t)$ and $A_{6}(x, y, t)$ are given by:

$$
\begin{gathered}
A_{1}(x, y, t)=\lim _{\Delta t \rightarrow 0} \iint\left(x-x_{1}\right)^{\gamma} \\
\frac{n\left(x-x_{1}, y-y_{1}, \Delta t\right)}{\Delta t^{\beta}} d x_{1} d y_{1}
\end{gathered}
$$




$$
\begin{gathered}
A_{2}(x, y, t)=\lim _{\Delta t \rightarrow 0} \iint\left(y-y_{1}\right)^{\gamma} \\
\frac{n\left(x-x_{1}, y-y_{1}, \Delta t\right)}{\Delta t^{\beta}} d x_{1} d y_{1} \\
A_{3}(x, y, t)=\lim _{\Delta t \rightarrow 0} \iint\left(x-x_{1}\right)^{2 \gamma} \\
\frac{n\left(x-x_{1}, y-y_{1}, \Delta t\right)}{2 \Delta t^{\beta}} d x_{1} d y_{1} \\
A_{4}(x, y, t)=A_{5}(x, y, t)=\lim _{\Delta t \rightarrow 0} \iint\left(x-x_{1}\right)^{\gamma} \\
\left(y-y_{1}\right)^{\gamma} \frac{n\left(x-x_{1}, y-y_{1}, \Delta t\right)}{\Delta t^{\beta}} d x_{1} d y_{1} \\
A_{6}(x, y, t)=\lim _{\Delta t \rightarrow 0} \iint\left(y-y_{1}\right)^{2 \gamma} \\
\frac{n\left(x-x_{1}, y-y_{1}, \Delta t\right)}{2 \Delta t^{\beta}} d x_{1} d y_{1}
\end{gathered}
$$

The only way to satisfy (12) is for the terms within curly brackets to vanish, leading to a fractional Fokker-Planck equation:

$$
\begin{gathered}
\frac{\partial^{\beta} n}{\partial t^{\beta}}-\frac{\partial^{\gamma}}{\partial x^{\gamma}}\left[A_{1} n\right]-\frac{\partial^{\gamma}}{\partial y^{\gamma}}\left[A_{2} n\right]-\frac{\partial^{2 \gamma}}{\partial x^{2 \gamma}}\left[A_{3} n\right]- \\
-\frac{\partial^{2 \gamma}}{\partial x^{\gamma} \partial y^{\gamma}}\left[A_{4} n\right]-\frac{\partial^{2 \gamma}}{\partial y^{\gamma} \partial x^{\gamma}}\left[A_{5} n\right]-\frac{\partial^{2 \gamma}}{\partial y^{2 \gamma}}\left[A_{6} n\right]=0
\end{gathered}
$$

where $\beta$ and $\gamma$ are the fractal exponents characterizing the traffic flow process and the road structure across which the agents navigate and interact. Note also that (18) contributes to the state-of-the-art in traffic modeling by accounting for various fractal characteristics of transport processes, which have not been addressed by current models [17][38]. Next, we discuss how the fractal dynamic game can be formulated.

\section{Optimal Fractal DYNAMiC GAMES}

Borrowing concepts from optimal control [4], we define the fractal dynamic game by considering a finite time horizon between an initial time $t_{i}$ and a final time $t_{f}$ over which each player minimizes a criterion of the following form:

$$
\begin{gathered}
J(t, x, y, n)=\min _{d(t) \in D} \int_{x_{\min }}^{x_{\max }} d x^{\gamma} \int_{y_{\min }}^{y_{\max }} d y^{\gamma} \int_{u_{\min }}^{u_{\max }} \\
\int_{t_{i}}^{t_{f}} c(u, x, y, n, \tau) P(u, \tau \mid x, y, n, d) n(x, y, \tau) d \tau^{\alpha} d u(19)
\end{gathered}
$$

where $c(u, x, y, n, \tau)$ is a strictly convex function that depends not only on the cost $u$, but also on the density of players $n(x, y, \tau)$ and where $P(u, \tau \mid x, y, n, d)$ is described by a master equation of the type presented in (6) and represents the conditional $\mathrm{PDF}$ of the costs to attain value $u$ at time $\tau$ given the state $(x, y)$ and the corresponding actions $d(t)$ of the density of players $n(x, y, \tau)$. Note that in (19) the dynamics of the players are assumed to follow not a classical Poisson process as in [25][26][28], but are in fact characterized by a power law between inter-event times. Thus, these dynamics should be described by a fractal stochastic differential equation. Moreover, in the context of traffic modeling, the cost function depends both on the speed at which an agent wants to move in order to minimize his/her travel time and on the traffic density $n(x, y, \tau)$. Following the lines of dynamic games, we assume that all players try to minimize the same type of criterion.
Let us now consider an infinitesimal time interval $\Delta t$ and split the optimization of the criterion over two intervals, $\left[t_{i}, t_{i}+\Delta t\right]$ and $\left[t_{i}+\Delta t, t_{f}\right]$, as shown below:

$$
\begin{aligned}
& J(t, x, y, n)=\min \underset{\substack{d(\tau) \in D \\
t_{i} \leq \tau \leq t_{i}+\Delta t}}{\int_{x_{\min }}^{x_{\max }}} d x^{\gamma} \int_{y_{\min }}^{y_{\max }} d y^{\gamma} \\
& \int_{u_{\min }}^{u_{\max }} d u \int_{t_{i}}^{t_{i}+\Delta t} d \tau^{\alpha} c(u, x, y, n, \tau) P(u, \tau \mid x, y, n, d)(20)
\end{aligned}
$$

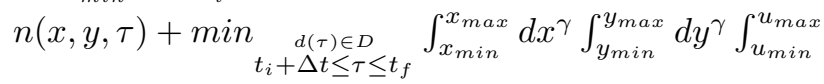

$$
\begin{aligned}
& \int_{t_{i}+\Delta t}^{t_{f}} c(u, x, y, n, \tau) P(u, \tau \mid x, y, n, d) n(x, y, \tau) d \tau^{\alpha} d u
\end{aligned}
$$

Assuming that the cost function $c(u, x, y, n, \tau)$ is continuous, we can approximate the first term in (20) by the next relation:

$$
\begin{aligned}
& \int_{t}^{t+\Delta t} c(u, x, y, n, \tau) P(u, \tau \mid x, y, n, d) n(x, y, \tau) d \tau^{\alpha}= \\
& \quad=c(u, x, y, n, t) P(u, t \mid x, y, n, d) \Delta t^{\alpha}+O\left(\Delta t^{\alpha}\right)
\end{aligned}
$$

where $O\left(\Delta t^{\alpha}\right)$ implies that infinitesimals of order higher than $\Delta t^{\alpha}$ are neglected. The fractal analysis of various cost functions shown in Fig. 1.c makes us to adopt a fractional differentiation $\Delta t^{\alpha}$ of order $\alpha$.

Now, we assume that the function $J\left(t_{i}+\Delta t, x, y, n\right)$ can be expanded in terms of fractional derivatives by means of the Taylor formula as follows:

$$
\begin{gathered}
J\left(t_{i}+\Delta t, x, y, n\right)=J\left(t_{i}, x, y, n\right)+\frac{\partial^{\alpha} J}{\partial t^{\alpha}} \Delta t^{\alpha}+\frac{\partial^{\gamma} J}{\partial x^{\gamma}} \Delta x^{\gamma} \\
+\frac{\partial^{\gamma} J}{\partial y^{\gamma}} \Delta y^{\gamma}+\frac{\partial^{2 \gamma} J}{\partial x^{2 \gamma}} \Delta x^{2 \gamma}+\frac{\partial^{2 \gamma} J}{\partial y^{2 \gamma}} \Delta y^{2 \gamma}+ \\
+\frac{\partial^{2 \gamma} J}{\partial x^{\gamma} \partial y^{\gamma}} \Delta x^{\gamma} \Delta y^{\gamma}+O\left(\Delta t^{\alpha}\right)+O\left(\Delta x^{2 \gamma}, \Delta y^{2 \gamma}\right)
\end{gathered}
$$

where all the fractional derivatives (which can be particularized to classical integer order derivatives) are evaluated in $(t, x(t), y(t))$. Note that in writing the relation in (22), we assume that $J\left(t_{i}, x, y, n\right)$ is continuous and fractal and it does not need to be differentiable.

By substituting (21) and (22) in (20), dividing it by $\Delta t^{\alpha}$ and taking the limit $\lim _{\Delta t \rightarrow 0}$, we obtain a fractal HamiltonJacobi type of equation for the loss function $J\left(t_{i}, x, y, n\right)$ :

$$
\begin{gathered}
\frac{\partial^{\alpha} J}{\partial t^{\alpha}}+B_{1}(t, x) \frac{\partial^{\gamma} J}{\partial x^{\gamma}}+B_{2}(t, y) \frac{\partial^{\gamma} J}{\partial y^{\gamma}}+B_{3}(t, x) \frac{\partial^{2 \gamma} J}{\partial x^{2 \gamma}}+ \\
B_{4}(t, y) \frac{\partial^{2 \gamma} J}{\partial y^{2 \gamma}}+B_{5}(t, x, y) \frac{\partial^{2 \gamma} J}{\partial x^{\gamma} \partial y^{\gamma}}+\min _{d(t) \in D} \\
\int_{u_{\min }}^{u_{\max }} c(u, x, y, n, \tau) P(u, \tau \mid x, y, n, d) d \tau^{\alpha} d u=0
\end{gathered}
$$

where the funtional coefficients $B_{1}(t, x), B_{2}(t, y), B_{3}(t, x)$, $B_{4}(t, y)$ and $B_{5}(t, x, y)$ satisfy the following relations:

$$
\begin{gathered}
\int_{x_{\min }}^{x_{\max }} d x^{\gamma} \int_{y_{\min }}^{y_{\max }} d y^{\gamma} \int_{u_{\min }}^{u_{\max }} d u \int_{t_{i}}^{t_{f}}\left(x_{t+\Delta t}-x_{t}\right)^{\gamma} \\
P\left(u, t \mid x_{t}, y_{t}, n, d\right) n\left(x_{t}, y_{t}, t\right) d t^{\alpha}=B_{1}(t, x) \Delta t^{\alpha}+O\left(\Delta t^{\alpha}\right) \\
\int_{x_{\min }}^{x_{\max }} d x^{\gamma} \int_{y_{\min }}^{y_{\max }} d y^{\gamma} \int_{u_{\min }}^{u_{\max }} d u \int_{t_{i}}^{t_{f}}\left(y_{t+\Delta t}-y_{t}\right)^{\gamma} \\
P\left(u, t \mid x_{t}, y_{t}, n, d\right) n\left(x_{t}, y_{t}, t\right) d t^{\alpha}=B_{2}(t, y) \Delta t^{\alpha}+O\left(\Delta t^{\alpha}\right) \\
\int_{x_{\min }}^{x_{\max }} d x^{\gamma} \int_{y_{\min }}^{y_{\max }} d y^{\gamma} \int_{u_{\min }}^{u_{\max }} d u \int_{t_{i}}^{t_{f}}\left(x_{t+\Delta t}-x_{t}\right)^{2 \gamma} \\
P\left(u, t \mid x_{t}, y_{t}, n, d\right) n\left(x_{t}, y_{t}, t\right) d t^{\alpha}=B_{3}(t, x) \Delta t^{\alpha}+O\left(\Delta t^{\alpha}\right) \\
P\left(u, t \mid x_{t}, y_{t}, n, d\right) n\left(x_{t}, y_{t}, t\right) d t^{\alpha}=B_{4}(t, y) \Delta t^{\alpha}+O\left(\Delta t^{\alpha}\right) \\
\int_{x_{\max }}^{x_{\max }} d x^{\gamma} \int_{y_{\min }}^{y_{\max }} d y^{\gamma} \int_{u_{\min }}^{u_{\max }} d u \int_{t_{i}}^{t_{f}}\left(y_{t+\Delta t}-y_{t}\right)^{2 \gamma}
\end{gathered}
$$




$$
\begin{gathered}
\int_{x_{\min }}^{x_{\max }} d x^{\gamma} \int_{y_{\min }}^{y_{\max }} d y^{\gamma} \int_{u_{\min }}^{u_{\max }} d u \int_{t_{i}}^{t_{f}}\left(x_{t+\Delta t}-x_{t}\right)^{\gamma} \\
(y(t+\Delta t)-y(t))^{\gamma} P\left(u, t \mid x_{t}, y_{t}, n, d\right) n\left(x_{t}, y_{t}, t\right) d t^{\alpha}= \\
=B_{5}(t, x, y) \Delta t^{\alpha}+O\left(\Delta t^{\alpha}\right)
\end{gathered}
$$

Note that we omit the subscripts $t$ for $x_{t}$ and $y_{t}$ because we assume that the variables $x$ and $y$ are evaluated at time $t$. Meanwhile, the density $n(x, y, t)$ is still described by the fractional Fokker-Planck type of equations:

$$
\begin{gathered}
\frac{\partial^{\beta} n(x, y, t)}{\partial t^{\beta}}-\frac{\partial^{\gamma}}{\partial x^{\gamma}}\left[A_{1} n(x, y, t)\right]- \\
-\frac{\partial^{\gamma}}{\partial y^{\gamma}}\left[A_{2} n(x, y, t)\right]-\frac{\partial^{2 \gamma}}{\partial x^{2 \gamma}}\left[A_{3} n(x, y, t)\right]- \\
-\frac{\partial^{2 \gamma}}{\partial x^{\gamma} \partial y^{\gamma}}\left[A_{4} n(x, y, t)\right]-\frac{\partial^{2 \gamma}}{\partial y^{\gamma} \partial x^{\gamma}}\left[A_{5} n(x, y, t)\right]- \\
-\frac{\partial^{2 \gamma}}{\partial y^{2 \gamma}}\left[A_{6} n(x, y, t)\right]=0
\end{gathered}
$$

The relations in (23) and (29) with prescribed final costs and intial conditions on $n(x, y, t)$ represent a fractal dynamic game. More precisely, (23) describes the backward evolution of agents decisions based on their future desideratum while (24) characterizes the forward evolution of the traffic based on its initial distribution.

\section{CONCLUSIONS AND FUTURE WORK}

In this paper, we defined a new fractal dynamic game by using finite-horizon optimal control theory and fractional (fractal) calculus concepts. The proposed fractal differential game facilitates the modeling of various human and biological networked processes such as road traffic or crowd evolution. By assuming that traffic agents are cost minimizers and by investigating the fractal structure of various real world processes, we set forth novel models for traffic behavior. Nevertheless, solving such a complex fractal dynamic game is a nontrivial task and requires future work because we need to develop efficient and robust numerical algorithms for the prediction of road traffic and financial market evolution.

Acknowledgements: Paul Bogdan thanks Sergio Pequito and Bruno Sinopoli of Carnegie Mellon University for their insightful comments on the topic of mean field games.

\section{REFERENCES}

[1] R. M. Axelrod, The Evolution of Cooperation, Basic Books, 2006.

[2] A.-L. Barabási and R. Albert, "Emergence of scaling in random Networks", Science, 286, pp. 509-512, 1999.

[3] A. Barrat, M. Barthelemy, A. Vespignani, Dynamical Processes on Complex Networks, Cambridge University Press, Cambridge, 2009.

[4] T. Basar and P. Bernhard, $H^{\infty}$-Optimal Control and Related Minimax Design Problems: A Dynamic Game, Springer, 1995.

[5] M. Batty and P. Longley, Fractal Cities: A Geometry of Form and Function, Academic Press, 1994.

[6] A. Blank and S. Solomon, "Power laws in cities population, financial markets and internet sites (scaling in systems with a variable number of components)", Physica A, 287, 1-2, pp.279-288, 2000.

[7] P. Bogdan and R. Marculescu, "Statistical physics approaches for network-on-chip traffic characterization," 7th IEEE/ACM Intl. Conf. on Hardware/software Codesign and System Synthesis, 2009.

[8] Calfornia Department of Transportation, Available at Caltrans PeMS website: http://pems.dot.ca.gov/?redirect=

[9] S. I. Denisov, W. Horsthemke and P. Hanggi "Generalized FokkerPlanck equation: derivation and exact solutions",Eur. Phys. J., 2009.

[10] C. Dogbé, "Modeling Crowd Dynamics by the Mean-Field Limit Approach", Math. Comput. Modelling, 52, pp. 1506-1520, 2010.

[11] M. Eigen and R. Winkler, Laws of the Game: How the Principles of Nature Govern Chance, Princeton University Press - Science, 1993.
[12] R. Feistel and W. Ebeling, Evolution of Complex Systems: SelfOrganization, Entropy and Development, Kluwer Academic Publishers - Mathematics, 1989.

[13] D. Friedman, D. N. Ostrov, "Gradient dynamics in population games: Some basic results", Journal of Mathematical Economics, Mathematical Economics: Special Issue in honour of Andreu Mas-Colell, Part 1, Vol. 46, Issue 5, pp. 691-707, 20 September 2010.

[14] H. Haken, Information and Self-Organization:A Macroscopic Approach to Complex Systems, Springer - Computers, 2006.

[15] D. Helbing, "A stochastic behavioral model and a 'microscopic' foundation of evolutionary game theory," Theory and Decision, 40, pp. 149-179, 1996.

[16] D. Helbing, "Microscopic foundation of stochastic game dynamical equations," in W. Leinfellner and E. Khler (eds.) Game Theory, Experience, Rationality, Kluwer Academic, Dordrecht, 1998.

[17] D. Helbing, "Traffic and related self-driven many-particle systems," Reviews of Modern Physics, vol. 73, Issue 4, pp. 1067-1141, 2001.

[18] R. Hilfer, Applications of Fractional Calculus in Physics, World Scientific, 2000.

[19] J. Hofbauer and K. Sigmund, Evolutionary Games and Population Dynamics, Cambridge University Press - Mathematics, 1998.

[20] A. L. Goldberger, L. A. N. Amaral, J. M. Hausdorff, P. Ch. Ivanov, C.-K. Peng, and H. Eugene Stanley, "Fractal Dynamics in Physiology: Alterations with Disease and Aging," Proc Natl Acad Sci USA, 99(Suppl 1), pp. 24662472, 2002.

[21] D. A. Gomes, J. Mohr, R. R. Souza, "Discrete time, finite state space mean field games", Journal de Mathematiques Pures et Appliques, Vol. 93, Issue 3, pp. 308-328, March 2010.

[22] O. Guéant, "A Reference Case for Mean Field Games Models", Journal de Mathèmatiques Pures et Appliquèxs, pp. 276-294, Vol. 92, Issue 3, September 2009.

[23] O. Guéant, J.-M. Lasry and P.-L. Lions, "Mean Field Games and Applications", in Paris-Princeton Lectures in Quantitative Finance, pp. Vol. 2003/2011, 205-266, 2011.

[24] A. Lachapelle, J. Salomon, and G. Turinici, "A monotonic algorithm for a mean field games model in economics", Math. Mod. Meth. Appl. Sci., 5(20), 2010.

[25] J.-M. Lasry and P.-L. Lions. "Jeux à champ moyen i. le cas stationnaire," C. R. Acad. Sci. Paris, 343(9), 2006.

[26] J.-M. Lasry and P.-L. Lions. "Jeux à champ moyen ii. horizon fini et contrôle optimal", C. R. Acad. Sci. Paris, 343(10), 2006.

[27] J.-M. Lasry and P.-L. Lions, "Mean field games", Japanese Journal of Mathematics, 2(1), March 2007.

[28] J.-M. Lasry, P.-L. Lions, and O. Guéant, "Application of Mean Field Games to Growth Theory", Technical report, CEREMADE, Dec. 2008.

[29] W. E. Leland, M. S. Taqqu, W. Willinger, and D. V. Wilson, "On the self-similar nature of ethernet traffic (extended version)," IEEE/ACM Trans. Netw., 2, 1, Feb. 1994.

[30] R. D. Luce and H. Raiffa,Games and Decisions: Introduction and Critical Survey, Dover, New York, 1957.

[31] B. B. Mandelbrot, The Fractal Geometry of Nature, Macmillan, 1983.

[32] B. B. Mandelbrot, Fractals and Scaling in Finance: Discontinuity, Concentration, Risk, Springer Quantitative Finance, 1997.

[33] J. F. Jr. Nash, "The Bargaining Problem," Econometrica, 18, 1950.

[34] J. von Neumann and O. Morgenstern,Theory of Games and Economic Behavior, Princeton, NJ. Princeton Univ. Press., 1947.

[35] H. Pauli, "Probleme der Modernen Physik," in Debye (ed.), Hirzel, Leipzig, 1928.

[36] A. D. Polyanin and A. V. Manshirov, Handbook of Mathematics for Engineers and Scientists, Taylor and Francis Group, 2007.

[37] A. Rapoport and A. M. Chammah, Prisoner's Dilemma, University of Michigan Press - Mathematics, 1965.

[38] H. Rehborn, S. L. Klenov, and J. Palmer, "Common Traffic Congestion Features studied in USA, UK, and Germany employing Kerner's Three-Phase Traffic Theory", in Physics and Society, Dec. 2010.

[39] B. M. Smirnov, Principles of Statistical Physics, Wiley, Berlin, 2006.

[40] G. Szabó and Fáth, "Evolutionary Games on Graphs," Phys. Rep., 446, pp. 97-216, 2007.

[41] M. C. Wang and G. E. Uhlenbeck, "On the Theory of the Brownian Motion II", Rev. Mod. Phys., 17, pp. 323342, 1945.

[42] E.J. Wagenmakers, S. Farrell, and R. Ratcliff, "Estimation and Interpretation of $1 / \mathrm{f}^{\alpha}$ Noise in Human Cognition,"Psychon. Bull. Rev., 11, 2004. 